\title{
1 Chikungunya virus infections: Time to act, time to treat
}

2 Rana Abdelnabi, Johan Neyts*, Leen Delang

3

4 University of Leuven, Department of Microbiology and Immunology, Rega Institute for

5 Medical Research, Laboratory of Virology and Chemotherapy, B-3000 Leuven, Belgium.

6 *Corresponding author: Johan Neyts, Herestraat 49, 3000 Leuven, Belgium. Tel 7 +3216321105, email: Johan.Neyts@kuleuven.be.

8

9

10

11

12

13

14

15

16

17

18

19 
Chikungunya virus (CHIKV) is a re-emerging alphavirus that caused massive outbreaks of chikungunya fever in several countries and regions in Africa, Asia and more recently in Central and South America. An acute CHIKV infection is usually associated with fever and arthritis and it is rarely fatal. However, $15-60 \%$ of patients suffer from chronic polyarthralgia for weeks, months or even for several years after the acute infection. There are currently no vaccines or antivirals available for the prevention or treatment of CHIKV infections. The development of potent and safe antivirals for prophylaxis (e.g. during travel to CHIKV-endemic regions) and treatment of CHIKV infections is urgently needed. We here review some of the recently reported CHIKV inhibitors, both directly-acting and host-targeting compounds.

\section{Keywords}

Chikungunya virus; antivirals; therapy; prophylaxis. 


\section{Transmission and prevalence}

Chikungunya virus (CHIKV) is an arbovirus that belongs to the alphavirus genus of the Togaviridae family [1]. The virus is transmitted by the bite of female mosquitoes of the Aedes species, mainly Ae. aegypti and Ae. albopictus. CHIKV was first isolated in 1953 after an epidemic in Tanzania [2]. Later, CHIKV infections were reported in Thailand followed by several cases in other Asian countries (1958-1973) [3]. In 2004, CHIKV re-emerged in Kenya after which massive epidemics occurred on several islands in the Indian Ocean such as La Réunion as well as in India [2]. During these epidemics, local transmission was also reported in a number of European countries such as Italy and France [2]. The most significant event responsible for the expansion of CHIKV infections was the genetic adaptation of CHIKV to the Ae. albopictus mosquito through an alanine-to-valine substitution at position 226 in the E1 surface glycoprotein gene (A226V)[4]. This adaptive mutation enhances the infectivity and transmission of the virus by Ae. albopictus [4]. The fact that Ae. albopictus thrives both in tropical and in temperate regions played a key role in the rapid spread of CHIKV in this period. In December 2013, the first local transmission of CHIKV was reported in the Americas on the Saint Martin Island. From there, it rapidly spread to the rest of the region. Until today, more than one million cases of CHIKV infection (www.who.int) have been reported in the Caribbean region and countries of Central and South America [2].

\section{Clinical impact}

The acute disease caused by CHIKV infection is characterized by a rapid onset of fever and by arthralgia resulting in debilitating pain similar to that of rheumatoid arthritis [1]. The acute disease caused by CHIKV infections is self-limiting and symptoms mostly resolve within 1 to 2 weeks [5]. However, 15-60\% of patients develop a chronic polyarthritis that 
can severely incapacitate the patient for weeks, months and even several years after the acute stage $[1,5]$.

Recently, CHIKV infections have been associated with neurological complications such as Guillain-Barré syndrome and meningoencephalitis [1,6*]. Other severe complications have been reported as well including conjunctivitis, myocarditis, pancreatitis and kidney failure [7]. During the La Réunion Island outbreak in 2006, CHIKV mother-to-child transmission in the perinatal period was reported for the first time [8]. Neonatal CHIKV infections have been implicated in meningoencephalitis and high morbidity rate. Recently, the first case of neonatal CHIKV-induced encephalitis has been reported in Brazil [9]. Due to the high socio-economic impact of its infections, CHIKV is now considered a real health threat to tropical and temperate areas colonized by Aedes mosquitoes.

\section{Replication cycle}

CHIKV enters the host cell by receptor-mediated endocytosis. Within the endosome, the viral envelope glycoprotein E1 undergoes conformational changes at low $\mathrm{pH}$ followed by fusion with the endosomal membrane [10*]. As a result, the viral nucleocapsid is released into the cytoplasm and then disassembled to release the viral RNA genome. The viral genome is then translated by the host cell machinery to generate the non-structural polyprotein which is processed to yield the $\mathrm{P} 123$ precursor and free nsP4 protein (the viral RNA-dependent RNA polymerase). The P123 precursor, nsP4 and host proteins together form an initial replication complex which synthesizes the negative-strand RNA [10*]. The negative-strand RNA is used as a template for synthesis of positive-strand genomic RNA and subgenomic RNA (26S RNA). The 26S RNA serves as the mRNA for the synthesis of the structural polyprotein [C-pE2-6K-E1]. After maturation of the envelope 
glycoproteins, the assembly of the nucleocapsids takes place in the cytoplasm followed by budding through the cell membrane where these nucleocapsids acquire the lipid bilayer envelope with the virus-encoded glycoproteins (Figure 1)[10*].

\section{Prevention and control}

The personal protective measures in endemic areas include wearing long-sleeved clothing, the use of insect repellant such as DEET or picaridin and mosquito nets [11]. For mosquito control, larvicides and insecticides are used. A novel strategy that is being explored in recent years is to control arbovirus transmission by infecting mosquitoes with the endosymbiotic bacterium Wolbachia. Introduction of the wMel strain of Wolbachia pipientis into Ae aegypti mosquitoes was shown to reduce the replication and transmission potential of several arboviruses including CHIKV [12], dengue virus [13], and Zika virus [14]. Infected mosquitoes could be released in endemic regions where they can invade the wild mosquito populations and thus reduce viral transmission (www.eliminatedengue.com).

Several strategies have been and are being studied for the development of CHIKV vaccines such as the use of inactivated, live attenuated, chimeric, virus-like particle, subunit protein, and DNA vaccines [15*]. Some vaccine candidates are currently being evaluated in preclinical and early phases of clinical trials but none of them have been approved yet $\left[15^{*}\right]$.

\section{Treatment}

Despite the worldwide (re-) emergence of CHIKV infections, there is no approved antiviral treatment available. Several classes of compounds that target either a viral or a host factor have been reported to inhibit CHIKV replication [10]. Although acute CHIKV infections are self-limiting, the use of potent antiviral(s) will reduce the viral load in infected patients, 
which may consequently (likely) reduce the chance to develop chronic infection. In addition, lowering the viral load in infected patients should also reduce the likelihood of transmission, for example in household settings. Here, we summarize some of the recently reported small molecule inhibitors of CHIKV replication (Figure 2).

\section{Directly-acting antivirals (DAAs)}

In theory, every step of the viral life cycle can be targeted by antiviral molecules (Figure 1). Some of the most successful antivirals that are being used to treat viral infections (such as those caused by HIV, hepatitis B virus (HBV), hepatitis C virus (HCV) and herpesviruses) are viral polymerase inhibitors [16]. The influenza virus polymerase inhibitor favipiravir (T-705), approved in Japan, has been reported to inhibit the in vitro replication of different CHIKV isolates and also to protect CHIKV-infected AG129 mice from severe neurological disease [17]. T-705 is activated intracellularly to its ribofuranosyl 5'-triphosphate form, which is then believed to inhibit the viral RNAdependent RNA polymerase (RdRp) [18]. The suggested mechanisms of antiviral activity of T-705 include (a) chain termination of the nascent viral RNA strand and/or (b) the induction of lethal mutagenesis in the viral genome [18]. Low-level T-705-resistant CHIKV variants could be selected in cell culture; a K291R mutation in motif F1 of the viral RdRp was shown to be responsible for the resistant phenotype [17].

We reported recently on a novel series of small molecules (the MADTP series) that efficiently inhibit the in vitro replication of both CHIKV lab strains and clinical isolates [19]. MADTP-resistant variants were identified to carry a proline-to-serine mutation (P34S) in the nsP1 gene [20**]. The nsP1 protein exerts guanine-7-methyltransferase and guanylyl-transferase activities which are required for capping and methylation of the newly synthesized viral RNA. Biochemical assays using the nsP1 protein of the related 
Venezuelan equine encephalitis virus (VEEV) revealed that compounds belonging to the MADTP series are specific inhibitors of the nsP1 guanylyl-transferase activity [20**]. The fact that some inhibitory activity was also observed against other alphaviruses (such as VEEV) in cell culture indicates that nsP1 might be a good target for the development of pan-alphavirus inhibitors.

Another potential molecular target for the development of DAA is the CHIKV nsP2, a multifunctional protein that possesses RNA helicase, nucleoside triphosphatase and RNA triphosphatase activity within its N-terminal domain and protease activity (required for processing of the non-structural viral polyprotein) at its C-terminal domain [21]. In addition, nsP2 is involved in shutting off host cell mRNA transcription and translation and inhibits cellular antiviral response [21]. Protease inhibitors are currently being successfully used to treat infections with HIV [22] and HCV [23]. The CHIKV nsP2 protease (a cysteine protease [24]) may hence be a relevant and excellent antiviral target. A series of CHIKV nsP2 protease inhibitors that had been designed in silico were shown to inhibit the nsP2 protease activity in cell-free enzymatic assays and the in vitro CHIKV replication in BHK-21 cells (with $\mathrm{EC}_{50}$ in the low $\mu \mathrm{M}$ range)[25*].

\section{Host-targeting antivirals (HTAs)}

As for all viruses, CHIKV depends on its host cell for virtually all steps in its infection cycle. Recently, several research strategies have identified host-virus interactions and host functions that are critical for viral replication. Targeting host factors instead of viral proteins is an attractive yet somehow also controversial strategy for antiviral intervention. On the one hand, the likelihood that drug-resistant variants will emerge is typically lower than in case of DAAs. Furthermore, the chance that such HTA exerts panalphavirus activity may be higher. On the other hand, inhibiting the primary functions of the host factors may possibly result in toxicity/adverse effects. Feasibility of such antiviral 
strategies will likely depend on which host factor is targeted and how the virus and the cell depend on its function(s). Example of HTAs against other viruses are the HIV entry inhibitor Maraviroc (a CCR5 receptor antagonist) [26] and the cyclophilin-targeting compound Alisporivir that was shown to be a highly potent inhibitor of HCV replication both in vitro and in infected patients [27].

A potential class of HTA CHIKV antivirals may be bryostatin analogs. Bryostatin 1 is a potent protein kinase $\mathrm{C}(\mathrm{PKC})$ modulator that has been explored in clinical trials for the treatment of cancer, Alzheimer's disease and for the eradication of HIV reservoirs [28]. PKC is a family of serine/threonine kinases that regulate many cellular processes such as proliferation and apoptosis. Unlike phorbol esters, which are also PKC activators, bryostatin has no tumor-promoting effect. Recently, a series of salicylate-based bryostatin analogs were reported to inhibit the CHIKV replication in the low $\mu \mathrm{M}$ range [28]. Interestingly, analogs that were designed not to (or less efficaciously) bind to PKC isoforms (by attaching a "cap" to the scaffold) still inhibited CHIKV replication [28]. Combination with PKC inhibitors counteracted the antiviral activity of a non-capped analog but did not affect that of capped analogs, providing further evidence that the antiCHIKV activity of the latter is PKC-independent [29].

Berberine is a plant-derived alkaloid that was reported to inhibit the in vitro replication of different CHIKV strains and to reduce the viral load and joints inflammation in CHIKVinfected mice [30*]. Interestingly, berberine was shown to reduce the activation of the major mitogen-activated protein kinase (MAPK) signaling pathways in CHIKV-infected cells [30*]. These pathways were found to be activated during CHIKV infection and to be important for the formation of infectious virus particles.

Another target to be blocked by HTAs could be the entry of CHIKV into the host cell (by for example flavaglines [31], obatoclax [32] and others (reviewed in [33]). Chloroquine, 
which inhibits CHIKV entry in vitro by blocking acidification of endosome, is the only (entry) inhibitor that was evaluated so far in a clinical study. However, this study yielded disappointing results [34]. Other potential targets for HTAs include the inhibition of heat shock protein 90 (HSP-90) [35] and cellular furins [36].

\section{Conclusion}

The worldwide re-emergence of CHIKV and the high morbidity rate associated with CHIKV infections calls for action. Besides vector control and prevention, another pillar of the CHIKV action plan should be the development of potent antiviral compounds. During acute CHIKV infections, potent antivirals should be able to reduce viral load in infected patients which will as a consequence decrease the severity of the symptoms. As the period of viremia is rather short, treatment should be started quickly after disease onset. A reduction of the viral load may also be expected to decrease the transmission efficiency of the virus by mosquitoes [37]. As the likelihood to develop chronic CHIKV disease is higher in persons with more severe symptoms during the acute phase of infection [38], administration of a potent antiviral during the acute infection may hopefully decrease the chances to develop chronic disease. It remains however to be studied whether CHIKV inhibitors may have a beneficial effect on the development of chronic arthritis. In chronically infected patients and macaques CHIKV RNA is detected in the synovial fluid in the joints even months after the infection [39,40]. It has however not yet been unequivocally demonstrated whether this represents truly replicating virus or rather the presence of defective viral genomic RNA. Potent and safe CHIKV inhibitors may potentially also be used as household-prophylaxis. Indeed, the probability of CHIKV transmission was shown to be $12 \%$ between household members but dropped to $0.3 \%$ for those living $50 \mathrm{~m}$ away [41**]. 
So far a number of classes of compounds (either targeting a viral or a host factor) have

207

208 been reported to inhibit the in vitro CHIKV replication. In most cases, the mechanism of the antiviral activity has not been elucidated and none of these molecules has progressed towards further development. The CHIKV RdRp and nsP2 protease may be promising targets for antiviral therapy. The viral guanylyl-transferase (encoded by nsP1) has also been shown to be an excellent target for the inhibition of alphavirus replication. Interestingly, the viral RNA polymerase inhibitor favipravir, licensed in Japan for the treatment of influenza virus infections, inhibits CHIKV replication in vitro and in a mouse model. Possibly the potential use of this compound in CHIKV-infected patients could be explored. Finally, since some alphaviruses such as the Venezuelan equine encephalitis virus could be potentially used as bio-weapons, it may be advisable to broaden the p123 to the development of inhibitors with pan-alphavirus activity.

Currently highly potent drugs are only available for the treatment of a limited number of viruses. However, with sufficient time and effort, it should also be possible to develop safe and potent antiviral molecules for the treatment and/or prophylaxis of alphavirus infections.

\section{References and recommended reading}

Papers of particular interest, published within the period of review, have been highlighted as:

\section{* of special interest}

** of outstanding interest 


\section{References}

1. Morrison CR, Plante KS, Heise MT: Chikungunya Virus: Current Perspectives on a Reemerging Virus. Microbiol Spectr. 2016, 4:143-161.

2. Presti A Lo, Cella E, Angeletti S, Cic- M: Molecular epidemiology, evolution and phylogeny of Chikungunya virus: an updating review. Infect. Genet. Evol. 2016, 41:270-278.

3. Weaver SC, Forrester NL: Chikungunya: Evolutionary history and recent epidemic spread. Antiviral Res. 2015, 120:32-39.

4. Amraoui F, Failloux A-B: Chikungunya: an unexpected emergence in Europe. Curr. Opin. Virol. 2016, 21:146-150.

5. Mccarthy MK, Morrison TE: Chronic chikungunya virus musculoskeletal disease : what are the underlying mechanisms ? Futur. Microbiol. 2016, 11:331-334.

6*. Gérardin P, Couderc T, Bintner M, Tournebize P, Renouil M, Lémant J, Boisson V, Borgherini G, Staikowsky F, Schramm F, et al.: Chikungunya virus-associated encephalitis: A cohort study on La Réunion Island, 2005-2009. Neurology 2016, 86:94-102.

7. Thiberville SD, Moyen N, Dupuis-Maguiraga L, Nougairede A, Gould E a., Roques P, de Lamballerie X: Chikungunya fever: Epidemiology, clinical syndrome, pathogenesis and therapy. Antiviral Res. 2013, 99:345-370.

8. Ramful D, Carbonnier M, Pasquet M, Bouhmani B, Ghazouani J, Noormahomed T, Beullier G, Attali T, Samperiz S, Fourmaintraux A, et al.: Mother-to-child 
transmission of Chikungunya virus infection. Pediatr Infect Dis J 2007, 26:811815.

9. Bandeira AC, Campos GS, Sardi SI, Rocha VFD, Rocha GCM: Neonatal encephalitis due to Chikungunya vertical transmission: First report in Brazil. IDCases

10*. Abdelnabi R, Neyts J, Delang L: Towards antivirals against chikungunya virus. Antiviral Res. 2015, 121:59-68.

11. Patterson J, Sammon M, Garg M: Dengue, Zika and Chikungunya: Emerging Arboviruses in the New World. West. J. Emerg. Med. 2016, 17:671-679.

12. Aliota MT, Walker EC, Uribe Yepes A, Dario Velez I, Christensen BM, Osorio JE: The

14. Aliota MT, Peinado SA, Velez ID, Osorio JE, Campos GS, Bandeira AC, Sardi SI, Camacho E, Paternina-Gomez M, Blanco PJ, et al.: The wMel strain of Wolbachia Reduces Transmission of Zika virus by Aedes aegypti. Sci. Rep. 2016, 6:28792.

15*. Smalley C, Erasmus JH, Chesson CB, Beasley DWC: Status of research and development of vaccines for chikungunya. Vaccine 2016, 34:2976-2981.

16. Tsai C-H, Lee P-Y, Stollar V, Li M-L: Antiviral therapy targeting viral polymerase. Curr. Pharm. Des. 2006, 12:1339-1355. 
17. Delang L, Segura Guerrero N, Tas A, Quérat G, Pastorino B, Froeyen M, Dallmeier K, Jochmans D, Herdewijn P, Bello F, et al.: Mutations in the chikungunya virus non-structural proteins cause resistance to favipiravir ( $\mathrm{T}-705)$, a broadspectrum antiviral. J. Antimicrob. Chemother. 2014, doi:10.1093/jac/dku209.

18. Furuta Y, Gowen BB, Takahashi K, Shiraki K, Smee DF, Barnard DL: Favipiravir (T-705), a novel viral RNA polymerase inhibitor. Antiviral Res. 2013, 100:446454.

19. Gigante A, Canela M-D, Delang L, Priego E-M, Camarasa M-J, Querat G, Neyts J, Leyssen P, Pérez-Pérez M-J: Identification of [1,2,3]triazolo[4,5-d]pyrimidin$7(6 \mathrm{H})$-ones as novel inhibitors of Chikungunya virus replication. J. Med. Chem. 2014, 57:4000-8.

20**. Delang L, Li C, Tas A, Quérat G, Albulescu IC, De Burghgraeve T, Guerrero NAS, Gigante A, Piorkowski G, Decroly E, et al.: The viral capping enzyme nsP1: a novel target for the inhibition of chikungunya virus infection. Sci. Rep. 2016, 6:31819.

21. Fros JJ, van der Maten E, Vlak JM, Pijlman GP: The C-terminal domain of chikungunya virus nsP2 independently governs viral RNA replication, cytopathicity, and inhibition of interferon signaling. J. Virol. 2013, 87:10394400.

22. Walmsley S: Protease inhibitor-based regimens for HIV therapy: safety and efficacy. J. Acquir. Immune Defic. Syndr. 2007, 45 Suppl 1:S5-13; quiz S28-31.

23. Berman K, Kwo PY: Boceprevir, an NS3 Protease Inhibitor of HCV. Clin. Liver Dis. 2009, 13:429-439. 
24. Rausalu K, Utt A, Quirin T, Varghese FS, Žusinaite E, Das PK, Ahola T, Merits A:

Chikungunya virus infectivity, RNA replication and non-structural polyprotein processing depend on the nsP2 protease's active site cysteine residue. Sci. Rep. 2016, 6:37124.

25*. Das PK, Puusepp L, Varghese FS, Utt A, Ahola T, Kananovich DG, Lopp M, Merits A, Karelson M: Design and validation of novel chikungunya virus protease inhibitors. Antimicrob. Agents Chemother. 2016, 7945:AAC.01421-16.

26. Lieberman-Blum SS, Fung HB, Bandres JC: Maraviroc: A CCR5-receptor antagonist for the treatment of HIV-1 infection. Clin. Ther. 2008, 30:12281250.

27. Zeuzem S, Flisiak R, Vierling JM, Mazur W, Mazzella G, Thongsawat S, Abdurakhmanov D, Van Kinh N, Calistru P, Heo J, et al.: Randomised clinical trial: alisporivir combined with peginterferon and ribavirin in treatmentnaive patients with chronic HCV genotype 1 infection (ESSENTIAL II). Aliment. Pharmacol. Ther. 2015, 42:829-844.

28. Staveness D, Abdelnabi R, Near KE, Nakagawa Y, Neyts J, Delang L, Leyssen P, Wender PA: Inhibition of Chikungunya Virus-Induced Cell Death by Salicylate-Derived Bryostatin Analogues Provides Additional Evidence for a PKC-Independent Pathway. J. Nat. Prod. 2016, doi:10.1021/acs.jnatprod.5b01017.

29. Abdelnabi R, Staveness D, Near KE, Wender PA, Delang L, Neyts J, Leyssen P: Comparative analysis of the anti-chikungunya virus activity of novel bryostatin analogs confirms the existence of a PKC-independent mechanism. 
Biochem. Pharmacol. 2016, 120:15-21.

30*. Varghese FS, Thaa B, Amrun SN, Simarmata D, Rausalu K, Nyman TA, Merits A, McInerney GM, Ng LFP, Ahola T: The antiviral alkaloid berberine reduces chikungunya virus-induced mitogen-activated protein kinase (MAPK) signaling. J. Virol. 2016, doi:10.1128/JVI.01382-16.

31. Wintachai P, Thuaud F, Basmadjian C, Roytrakul S, Ubol S, Désaubry L, Smith DR: Assessment of flavaglines as potential chikungunya virus entry inhibitors. Microbiol. Immunol. 2015, 59:129-141.

32. Varghese FS, Rausalu K, Hakanen M, Saul S, Kümmerer BM, Susi P, Merits A, Ahola T: Obatoclax inhibits alphavirus membrane fusion by neutralizing the acidic environment of endocytic compartments. Antimicrob Agents Chemother. 2016.

33. van Duijl-Richter MKS, Hoornweg TE, Rodenhuis-Zybert IA, Smit JM: Early events in chikungunya virus infection???from virus cell binding to membrane fusion. Viruses 2015, 7:3647-3674.

34. Chopra A, Saluja M, Venugopalan A: Effectiveness of chloroquine and inflammatory cytokine response in patients with early persistent musculoskeletal pain and arthritis following chikungunya virus infection. Arthritis Rheumatol. 2014, 66:319-326.

35. Rathore APS, Haystead T, Das PK, Merits A, Ng ML, Vasudevan SG: Chikungunya virus nsP3 \& nsP4 interacts with HSP-90 to promote virus replication: HSP90 inhibitors reduce CHIKV infection and inflammation in vivo. Antiviral Res. 2014, 103:7-16.

36. Ozden S, Lucas-Hourani M, Ceccaldi PE, Basak A, Valentine M, Benjannet S, 
Hamelin J, Jacob Y, Mamchaoui K, Mouly V, et al.: Inhibition of Chikungunya virus infection in cultured human muscle cells by furin inhibitors: Impairment of the maturation of the E2 surface glycoprotein. J. Biol. Chem. 2008, 283:21899-21908.

37. Pesko K, Westbrook CJ, Mores CN, Lounibos LP, Reiskind MH: Effects of Infectious Virus Dose and Bloodmeal Delivery Method on Susceptibility of Aedes aegypti and Aedes albopictus to Chikungunya Virus. J. Med. Entomol. $2009,46: 395-399$.

38. Sissoko D, Malvy D, Ezzedine K, Renault P, Moscetti F, Ledrans M, Pierre V: Postepidemic Chikungunya disease on reunion island: Course of rheumatic manifestations and associated factors over a 15-month period. PLoS Negl. Trop. Dis. 2009, 3.

39. Hoarau J-J, Jaffar Bandjee M-C, Krejbich Trotot P, Das T, Li-Pat-Yuen G, Dassa B, Denizot M, Guichard E, Ribera A, Henni T, et al.: Persistent chronic inflammation and infection by Chikungunya arthritogenic alphavirus in spite of a robust host immune response. J. Immunol. 2010, 184:5914-27.

40. Labadie K, Larcher T, Joubert C, Mannioui A, Delache B, Brochard P, Guigand L, Dubreil L, Lebon P, Verrier B, et al.: Chikungunya disease in nonhuman primates involves long-term viral persistence in macrophages. J. Clin. Invest. 2010, 120:894-906.

41**. Salje H, Lessler J, Paul KK, Azman AS, Rahman MW, Rahman M, Cummings D, Gurley ES, Cauchemez S: How social structures, space, and behaviors shape the spread of infectious diseases using chikungunya as a case study. Proc. 
Natl. Acad. Sci. 2016, 113:201611391.

368

369 


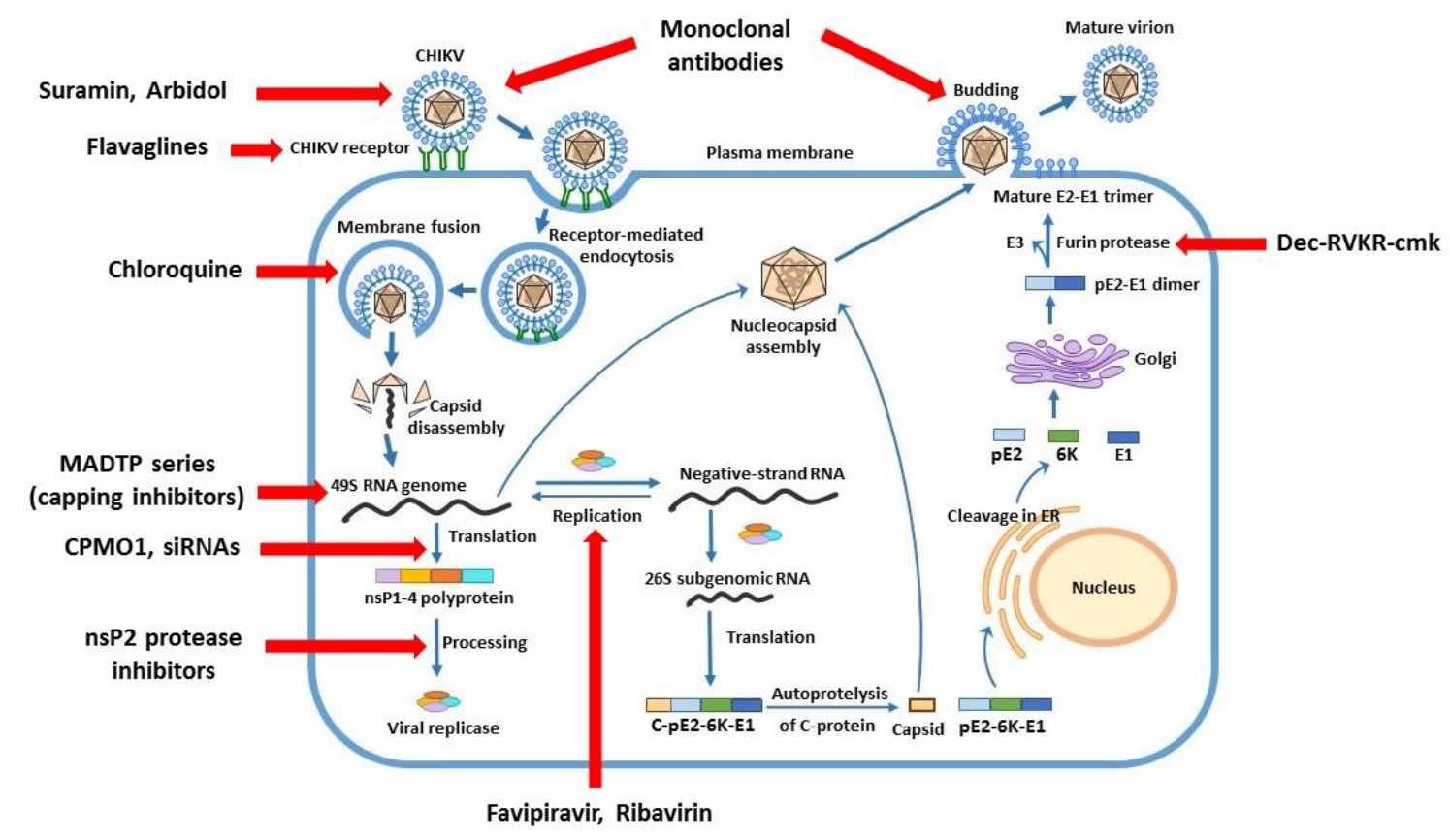

Figure. 1. Schematic representation of CHIKV replication cycle and the reported targets for

some CHIKV inhibitors. CHIKV enters the host cell by receptor-mediated endocytosis. The acidic environment within the endosome then triggers the fusion of the viral envelope with the endosomal membrane resulting in the release of the nucleocapsid into the cytoplasm. The nucleocapsid disassembles to liberate the viral genome which is being translated to produce the viral nonstructural polyprotein (nsP1-4). After proteolytic cleavage by nsP2, the viral nonstructural proteins form together the viral replicase which catalyzes the synthesis of a negative-sense RNA strand which serves as a template for synthesis of both the full-length positive-sense genome and the subgenomic (26S) RNA. The subgenomic RNA is being translated to produce the structural polyprotein (C-E3-E2-6K-E1) which is then cleaved to produce the individual structural proteins. Consequently, the new virus particle is assembled and released by budding out through the plasma membrane where it acquires its envelope with embedded viral glycoproteins. CPMO1 is synthetic phosphorodiamidate morpholino oligomer that inhibits protein synthesis. Dec-RVKR-cmk (decanoyl-RVKR-chloromethyl ketone) is cellular furin inhibitor. This figure is adapted from [10*], red arrows indicate an inhibitory effect. 
<smiles>NC(=O)c1nc(F)c[nH]c1=O</smiles>

Favipiravir (T-705)

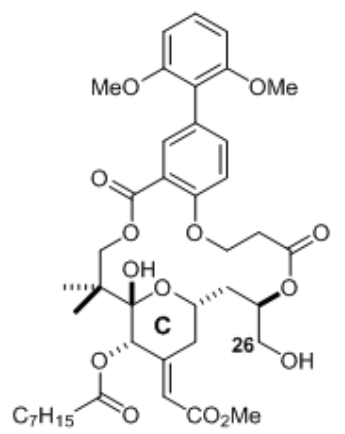

Non-capped bryostatin analog

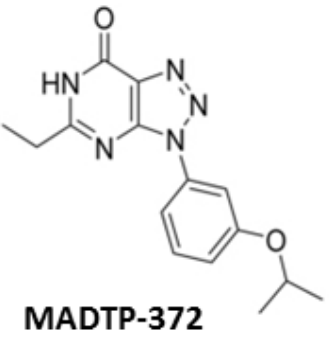<smiles>CCOC(=O)c1c(CSc2ccccc2)n(C)c2cc(Br)c(O)c(CN(C)C)c12</smiles>

Arbidol

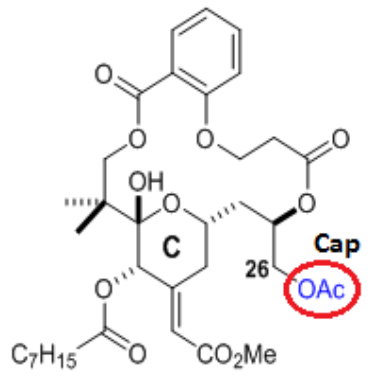

Capped bryostatin analog<smiles>COc1ccc2cc3[n+](cc2c1O)CCc1cc2c(cc1-3)OCO2</smiles>

Berberine

388 Figure. 2. Chemical structures of selected inhibitors of chikungunya virus replication. 